\title{
LIGHT SCATTERING TECHNIQUES AS A KEY IN PROVIDING INSIGHTS INTO THE INTERACTIONS AND STABILITY OF ALKALI-ACTIVATED MATERIALS BASED ON BLAST FURNACE SLAG
}

\author{
Michal KALINA, Petr HRUBÝ, Jiří SMILEK, Lukáš KALINA, Vlastimil BÍLEK Jr. \\ Brno University of Technology, Faculty of Chemistry, Brno, Czech Republic, EU, kalina-m@fch.vut.cz
}

https://doi.org/10.37904/nanocon.2021.4376

\begin{abstract}
Light scattering techniques represent a group of methods providing the absolute information on the molecular weight, particle size, conformation, polydispersity and the stability of analyzed dispersion systems. These techniques are primarily used for the characterization of biopolymers, synthetic polymers and nanoparticles. The utilization in the area of alkali-activated materials based on blast furnace slag is more challenging, mainly due to their highly alkaline nature and also due to the generally used high content of solid fractions dispersed in liquid media. This contribution was focused either on the optimization of sample preparation as well as on the definition of the optimal setting of measuring parameters to be able to properly use the electrophoretic light scattering (Zetasizer Nano ZS; Malvern Panalytical Ltd.) for the description of the stability of these complex samples. For these purposes, we studied dispersion systems of blast furnace slag in activating solutions (sodium hydroxide, sodium carbonate and sodium silicate). The obtained zeta potential provided crucial insight into the individual sample stability. The results are indicating that the proper technique of sample dispersion and the maintenance of the precise content and ratios of individual components represent important parameters managing the stability of alkali-activated materials based on blast furnace slag.
\end{abstract}

Keywords: Alkali-activated materials, blast furnace slag, conductivity, stability, zeta potential

\section{INTRODUCTION}

The development of modern building materials is nowadays closely connected to government restriction as well as by the environmental issues such as tendencies to reduce $\mathrm{CO}_{2}$ emissions and the efforts to utilize the waste materials and by-products from other industries to protect nature and natural raw materials [1]. Alkali-activated materials (AAM) represent the non-traditional cementitious materials, which fulfil these ideas and currently are studied as materials that could serve as the potential substitute to the ordinary Portland cement in some applications [2]. The typical example of AAM is alkali-activated blast furnace slag (AAS) [3]. Generally, these materials show interesting chemical [4,5] and temperature resistance [6] and high early strength [7] of the material but on the other hand the high autogenous and drying shrinkage must be mentioned as the main disadvantage of the broader practical application of AAM [8]. The published research also indicates the necessity to design a specific admixture exclusively for the individually used AAM to overcome this issue [9], otherwise, AAMs will never fulfil their potential and will never be competitive to Portland cement [10].

At this point, the need for a technique allowing the simple description of interactions among blast furnace slag (BFS) and other components in the systems (activation solutions, admixtures) seems to be crucial. One of the methods how to determine these characteristics is the method of electrophoretic light scattering allowing to determine the zeta potential of the electrokinetic potential in the interfacial double layer of BFS grains [11]. As can be found in the literature [12] this important parameter characterizing the surface chemistry of BFS is dependent on $\mathrm{pH}$, the nature of the used alkaline activator and also on the addition of admixture used for further processing of BFS dispersions. Moreover, the values of zeta potential determined for AAS are also 
influenced by the concentration of analysed particles. Kashani et al. [12] highlighted that the values of zeta potential of AAS have a direct connection to the rheological properties of slag-based suspensions containing sodium or potassium hydroxides and sodium silicate. The lowest yield stress was observed for slag suspension containing sodium silicate, followed by slag in water without activator, while it markedly increased for sodium hydroxide. Higher yield stress values are in accordance with the structure-making/structure-breaking theory of structural forces as well as with the determined values of zeta potential that were usually negative for slag in water, which was induced by the partial deprotonation of silanol groups on the slag surfaces. Contrary to alkali hydroxide activators, increasing dose of sodium silicate induced a shift to the more negative values of zeta potential and thus contribution of the repulsive electric double layer forces increased.

The zeta potential in cementitious suspensions can be determined using the electroacoustic method [13], enabling the measurement of concentrated suspensions [14], but an issue of too high alkalinity can still arise. In addition, often only the electrophoretic light scattering is available. However, it inherently requires a diluted sample, which may affect the results obtained. Therefore, our experimental work focused on optimizing the electrophoretic light scattering method for the investigation of the interactions and stabilization of BFS in various used AAS. For these purposes, we have used the dilution approach, where we studied the direct relation of BFS to $\mathrm{Na}^{+}$concentration and the total content of these components in the system to be able to predict the behaviour of original non-diluted samples.

\section{EXPERIMENTAL}

\subsection{Materials}

The individual samples of alkali-activated pastes were prepared using a blast furnace slag (BFS) with Blaine fineness of $400 \mathrm{~m}^{2} \cdot \mathrm{kg}^{-1}$ and density of $2.88 \mathrm{~g} \cdot \mathrm{cm}^{-3}$. The chemical composition of BFS as was obtained by Xray fluorescence analysis (XRF) is given in Table 1. The individual studied samples of alkali-activated slag (AAS) were prepared using the activation solutions of sodium hydroxide (Lach-Ner s.r.o), sodium carbonate (Lach-Ner, s.r.o) and sodium silicate having the molar ratio of $\mathrm{SiO}_{2} / \mathrm{Na}_{2} \mathrm{O}(\mathrm{Ms}=1.50)$ (Vodní sklo, a.s.).

Table 1 Chemical composition of blast furnace slag obtained by XRF analyses

\begin{tabular}{|c|c|c|c|c|c|c|c|c|c|c|}
\hline \multirow{2}{*}{ material } & \multicolumn{10}{|c|}{ Chemical composition (wt.\%) } \\
\cline { 2 - 22 } & $\mathrm{SiO}_{2}$ & $\mathrm{Al}_{2} \mathrm{O}_{3}$ & $\mathrm{CaO}$ & $\mathrm{MgO}$ & $\mathrm{SO}_{3}$ & $\mathrm{Na}_{2} \mathrm{O}$ & $\mathrm{K} 2 \mathrm{O}$ & $\mathrm{TiO}_{2}$ & $\mathrm{MnO}_{2}$ & $\mathrm{Fe}_{2} \mathrm{O}_{3}$ \\
\hline blast furnace slag & 34.7 & 9.1 & 41.1 & 10.5 & 1.4 & 0.4 & 0.9 & 1.0 & 0.6 & 0.3 \\
\hline
\end{tabular}

\subsection{Sample preparation}

Concentration series of all activating solutions were prepared in advance prior to their mixing with slag. The specific range of concentrations was given by their achievability with respect to the original composition of the activators: $0-5 \mathrm{~mol} \mathrm{Na} \cdot \cdot \mathrm{dm}^{-3}$ for sodium carbonate solution, $0-9.93 \mathrm{~mol} \mathrm{Na}^{+} \cdot \mathrm{dm}^{-3}$ for sodium silicate solution and $0-15 \mathrm{~mol} \mathrm{Na}^{+} \cdot \mathrm{dm}^{-3}$ for sodium hydroxide solution. Then each activating solution was mixed with slag (1:1 by volume) to obtain $10 \mathrm{~cm}^{3}$ of the whole suspension (paste) and homogenized with a spatula for 3 minutes. After another two minutes of rest, the paste was diluted by demineralized water in the range 1:10, 1:50, 1:100, $1: 250,1: 500$ and 1:1000 and immediately characterized by electrophoretic light scattering (section 2.4).

\subsection{Physico-chemical characterization of activation solutions}

The individual alkali-activated solutions were characterized through potentiometric $\mathrm{pH}$ determination using the Titrando 888 module equipped with a combined Unitrode electrode for a highly alkaline pH region with an integrated Pt1000 temperature sensor (Metrohm AG, Switzerland). The data shown further in the text represent the mean values of five repeated measurements (results in the form of mean values $\pm S D, n=5$ ). The data processing was performed using Tiamo software (version 2.5; Metrohm AG, Switzerland). 
Dynamic viscosity of all the used alkali-activated solutions was measured employing Höppler falling ball viscometer AMVn (Anton Paar GmbH, Austria). The data shown further in the text represent mean values of three repeated measurements (results in the form of mean values $\pm S D, n=3$ ).

\subsection{Zeta potential measurement}

The effect of the type of alkaline activation (sodium hydroxide, sodium carbonate, sodium silicate with Ms = 1.5), their concentration in the sample (range from 0 to $15 \mathrm{~mol} \cdot \mathrm{dm}^{-3}$ concentration of $\mathrm{Na}^{+}$) and the dilution ratio (dilution by volume with distilled water in the range of 1:10, 1:50, 1:100,1:250,1:500 and 1:1000) of the individual alkali-activated BFS dispersions was investigated by the method of electrophoretic light scattering using Zetasizer Nano ZS (Malvern Panalytical Ltd., UK). Simultaneously the average scattered light intensity, conductivity and the zeta potential of the suspensions were measured in three repeated measurements of three individually taken samples (results are presented in the form of mean values $\pm \mathrm{SD}, n=9$ ). The obtained data from ELS analysis were processed by the Zetasizer software (version 7.11; Malvern Panalytical Ltd., UK).

\section{RESULTS AND DISCUSSION}

The principal task of this work was the investigation of the effect of the type of used alkali-activated solution and their concentration in the sample on the interactions with BFS. For these purposes, the measurement of zeta potential using the method of electrophoretic light scattering was selected. This method is used for investigation of the surface chemistry of colloidal dispersions, description of their surface charge with the corresponding stability of particles against mutual aggregation and for investigation of the interactions among particles in the system [5]. Due to the experimental limitations (mainly the limits for the $\mathrm{pH}$, conductivity and concentration), it was not possible to study the sample directly, but we had to perform a dilution with distilled water to be able to subsequently extrapolate the zeta potential to the original samples. For these purposes, the dilution ratios between 1:50 and 1:1000 (by volume) were used.

\subsection{Physico-chemical characterization}

The physico-chemical characterization of all the used activation solutions by measurement of their $\mathrm{pH}$, conductivity and dynamic viscosity was realized as the initial experiments necessary for subsequent discussion of zeta potential results obtained on AAS in a broader context in Section 3.2. The results of the measured pH and dynamic viscosity for all the used activation solutions are shown in Figure 1.
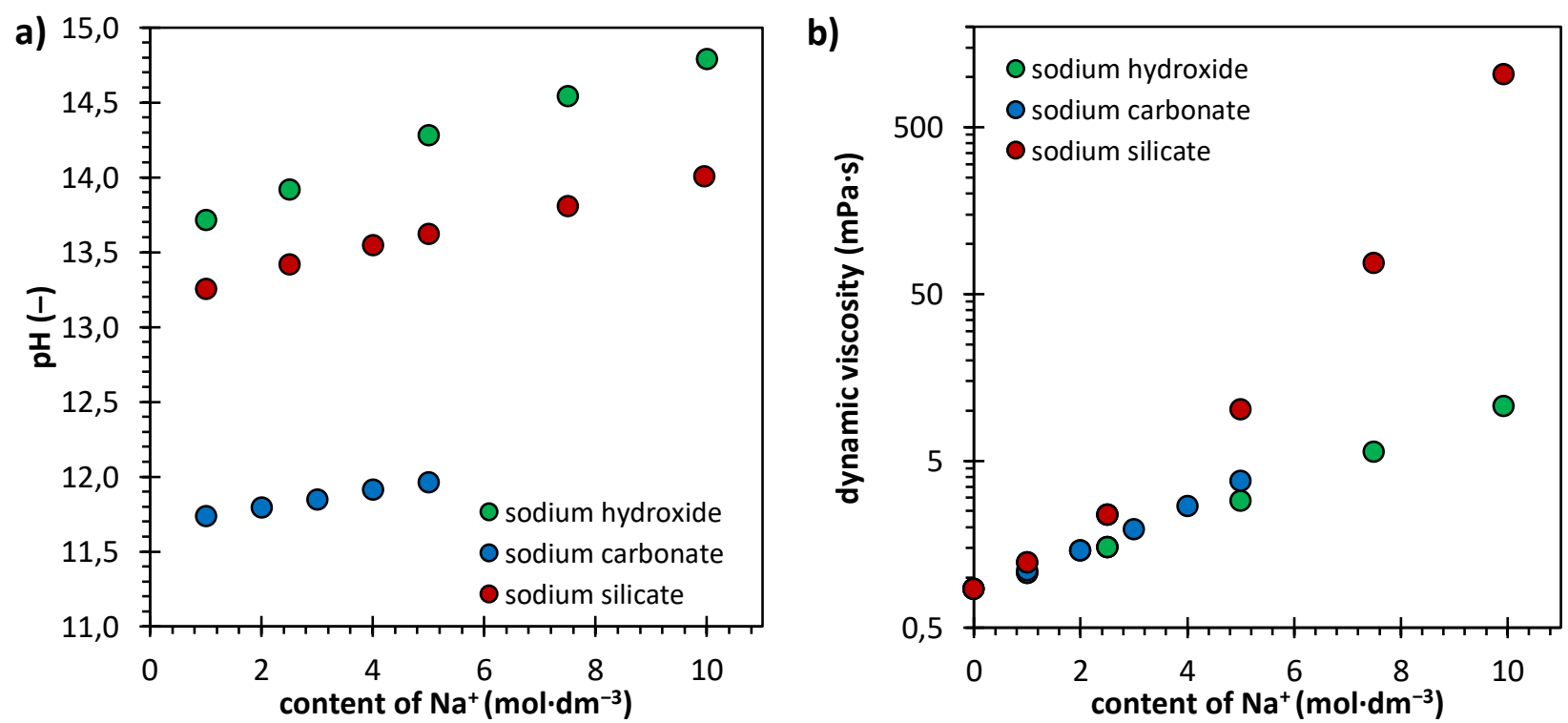

Figure $1 \mathrm{a}$ ) $\mathrm{pH}$ and b) dynamic viscosity of activation solutions recalculated on the real content of $\mathrm{Na}^{+}$ions 
The determined $\mathrm{pH}$ values are indicating expected dependences on the real content of $\mathrm{Na}^{+}$ions. Especially for sodium hydroxide solutions, the data need further corrections on activity coefficients. The highest $\mathrm{pH}$ values were measured for sodium hydroxide solutions and on the other hand in agreement with our expectation, the lowest $\mathrm{pH}$ values were measured for sodium carbonate solutions. The data from the measurement of dynamic viscosity of the individual activating solutions (Figure 1b) are indicating similar trends of sodium hydroxide and sodium carbonate (logarithm of dynamic viscosity has almost linear dependence on the concentration of $\mathrm{Na}^{+}$ in the solution). The different behaviour was observed in the sodium silicate activation solution, where the dependence of logarithm of dynamic viscosity on the concentration of $\mathrm{Na}^{+}$was much higher and indicated an almost polynomial trend in the data. These results are in good correlation with published literature [15].

\subsection{Zeta potential measurement}

The zeta potential measurement by using the method of ELS was used as the principal experimental approach for the investigation of the interactions and stability of dispersions of BFS in alkali-activated solutions (sodium hydroxide, sodium silicate and sodium carbonate). Due to the experimental limitations of ELS (pH and conductivity and concentration limits) the measurement was performed under the broad range of used dilution ratios (diluted by volume with distilled water 1:50, 1:100,1:250, 1:500 and 1:1000). For this reason, the lowest used dilution (1:10) was not reliably measured and thus the results are not shown.

a)

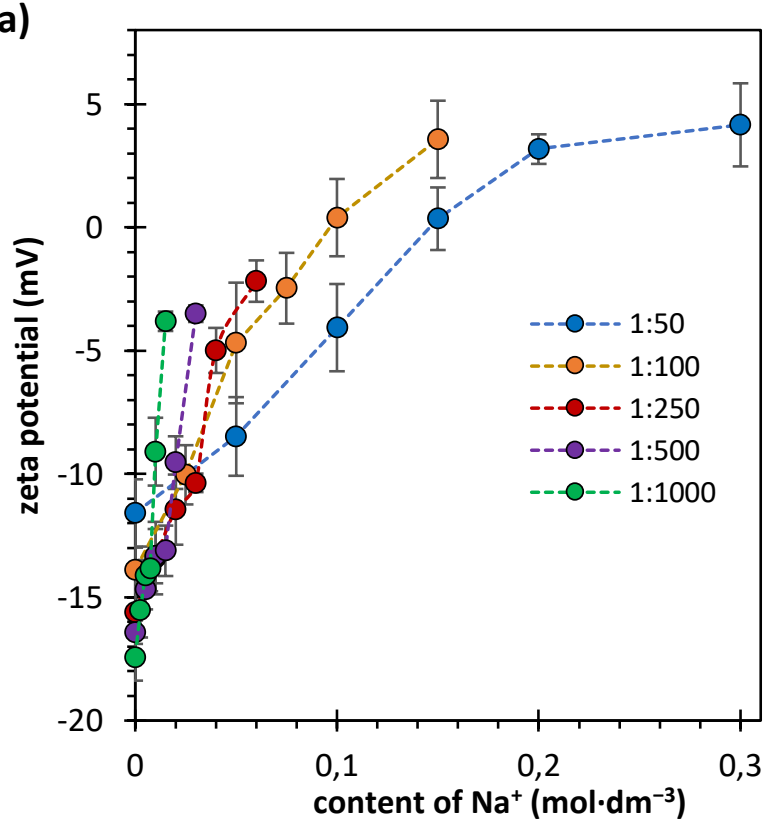

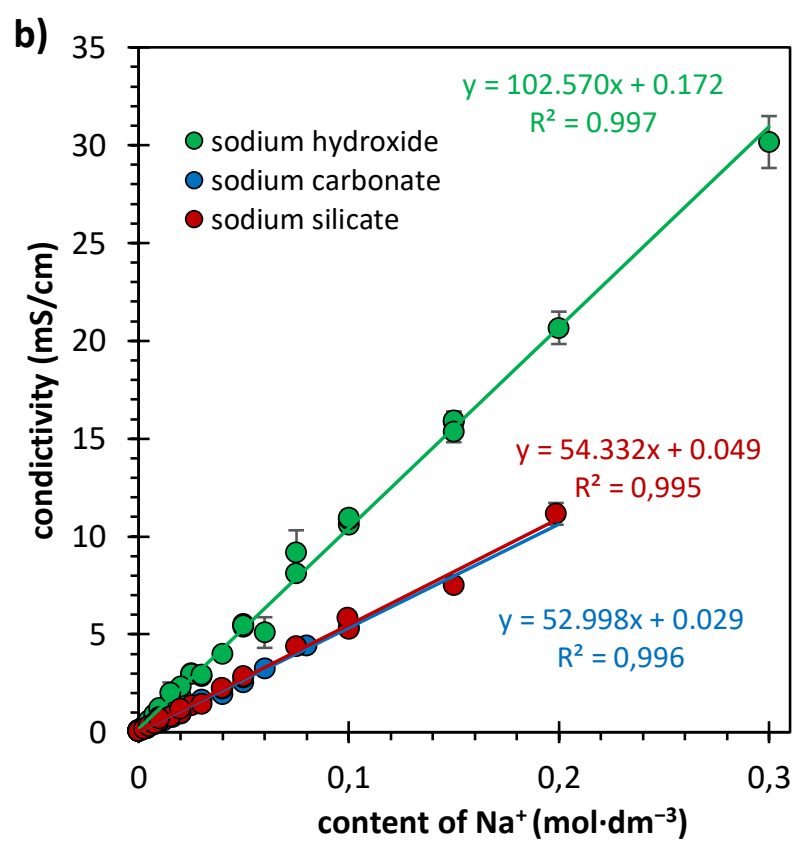

Figure 2 a) Development of zeta potential of various used dilution ratios of BFS dispersions in sodium hydroxide recalculated on the real content of $\mathrm{Na}+$ ion in the samples, b) Measured conductivities of BFS dispersions in all used alkaline activators recalculated on the real content of $\mathrm{Na}+$ ion in the samples.

The main task in this part of the experiments was the utilization of the dilution approach as the possibility to get an overview of the trend in zeta potential for individual samples in respect to the used dilution ratio, helping to predict and extrapolate the behaviour of original undiluted samples. The example of obtained zeta potential dependencies on the concentration of $\mathrm{Na}^{+}$ions in the samples as were obtained for sodium hydroxide alkaline activation for the individual used ranges of dilution are shown in Figure 2a. The results are indicating that the dilution has a minimal effect on the zeta potentials of BFS in distilled water without the addition of activators, where the average zeta potential $(-15.7 \pm 2.2) \mathrm{mV}$ was determined. With the increasing concentration of $\mathrm{Na}^{+}$ ions in the samples the difference from the original dispersions of BFS in the water started to be more

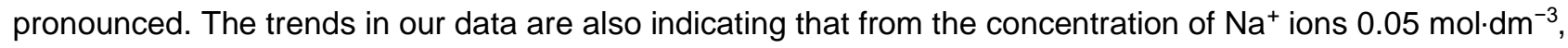


sample high conductivity and $\mathrm{pH}$ are starting to be the key features tailoring and affecting the final value of measured zeta potential of BFS dispersion. Figure $\mathbf{2} \mathbf{b}$ experimentally confirmed the direct relation of the concentration of $\mathrm{Na}^{+}$ions in BFS dispersions and its measured conductivity. Our dilution approach also indicated that at the concentration of $\mathrm{Na}^{+}$ions $0.1 \mathrm{~mol} \cdot \mathrm{dm}^{-3}$. BFS dispersions reached zero zeta potential, which is indicating their low electrostatic stabilization against aggregation. Further addition of $\mathrm{Na}^{+}$ions gradually shifted zeta potentials into the positive region of the scale. These results are in good agreement with published literature [12].

The comparison of two selected dilution ranges (1:50 and 1:1000 by volume) for the other two studied alkaline activators is shown in Figure 3. The presented data are indicating an opposite effect observed for the activation with sodium carbonate and sodium silicate in comparison to sodium hydroxide alkaline activation of BFS. In all studied dilution ranges, we have observed a significant shift to the more negative values of BFS dispersions zeta potential in comparison with original BFS dispersion in water. Again, this finding is in good correlation with published data [12]. This is indicating a more pronounced negative charge of individual particles in these samples with further addition of both these activating solutions and also an increase in their colloidal stability. These results are really interesting, especially considering the observed dependences of conductivities of BFS dispersions on $\mathrm{Na}^{+}$ion concentration showed in Figure $\mathbf{2 b}$.
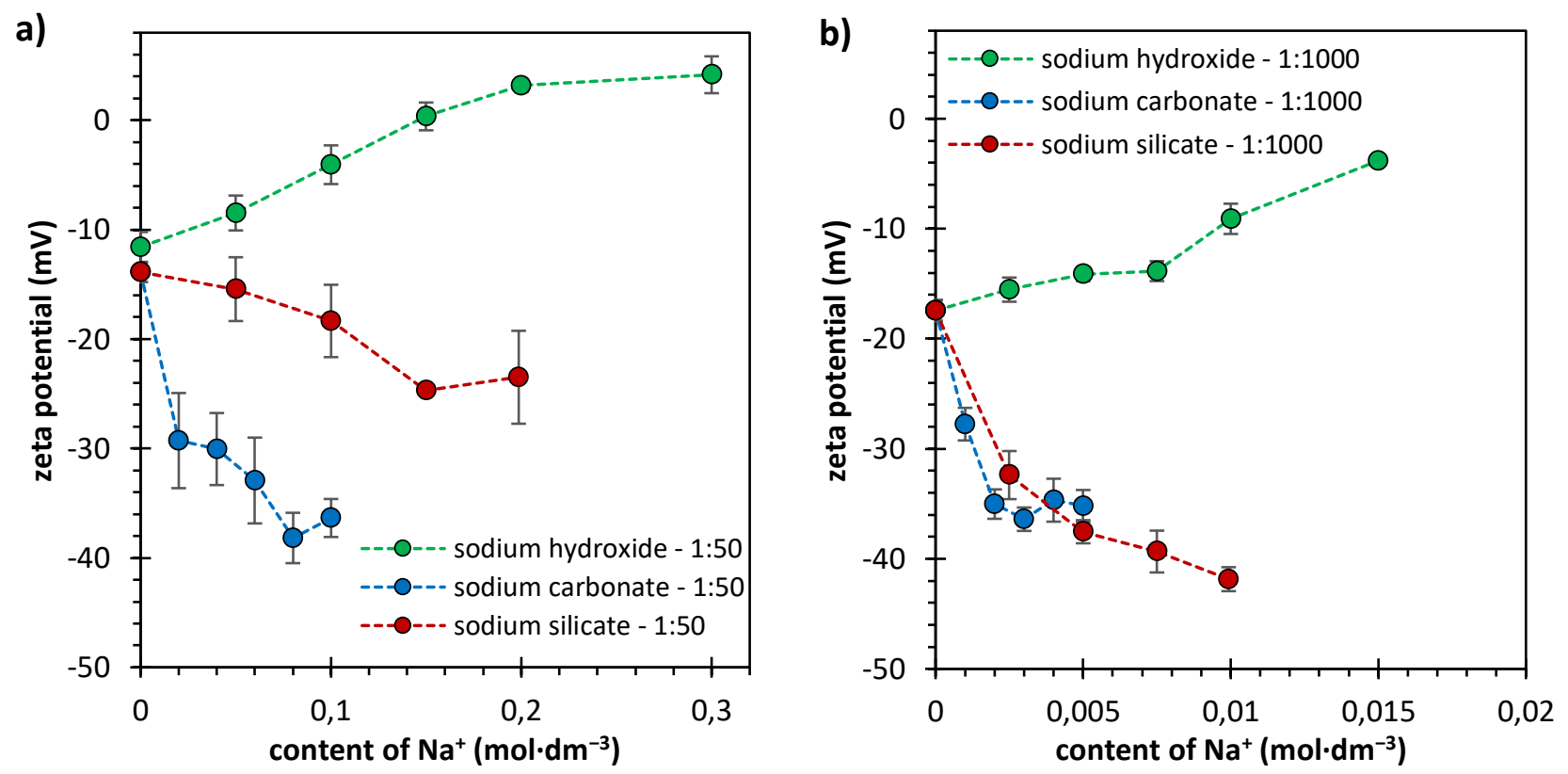

Figure 3 Development of zeta potential of BFS in used alkaline activators recalculated on the real content of $\mathrm{Na}+$ in the samples - a) 1:50 dilution (by volume) with water; b) 1:1000 dilution (by volume) with water

The conductivities of sodium silicate and sodium carbonate dispersions are showing similar trends as the data for sodium hydroxide. These experimental data are indicating a completely different mode of operation of sodium carbonate and sodium silicate compared to the classical BFS activation using sodium hydroxide. The literature $[9,12]$ explain this behaviour by the adsorption of carbonate or silicate anions onto the BFS grains.

The comparison of the dilution of sodium carbonate and sodium silicate is indicating an almost negligible effect in the case of sodium carbonate. This AAS showed the lowest $\mathrm{pH}$ in comparison with sodium silicate and sodium hydroxide solutions and due to this fact, the further dilution of these BFS dispersions has an almost negligible effect on the measured zeta potential and corresponding stability of these samples. On the other hand, sodium silicate solutions showed more alkaline $\mathrm{pH}$ and also significantly higher dynamic viscosity in comparison to sodium carbonate, which are probably the main reasons for the observed more significant role of dilution on the measured zeta potentials of BFS dispersions. 
Although the trend seems similar for both dilutions presented in Figure 3, lower dilution gives values closer to zero (for $\mathrm{NaOH}$ even to positive values) and it is thus questionable, what situation is in the highly concentrated suspensions prior to their dilution with higher ionic strength etc. Therefore, the results illustrate that the dilution extent together with the nature of the alkaline activator are crucial parameters for the obtained absolute values of zeta potential and one should be cautious when trying to predict the behaviour of the real concentrated suspension. The comparison of the values with those of concentrated suspensions could be very interesting but unfortunately has not been done due to the unavailability of the electroacoustic method able to measure concentrated suspensions $[11,13,14]$ at our department.

\section{CONCLUSION}

The main task of our work was the investigation of the effect of the type and the concentration of used alkaline activators on the stability and interactions between colloidal particles of BFS. For these purposes, we have used zeta potential as a crucial feature of analyzed samples together with the sample-dilution approach allowing us to predict further behaviour of these systems in a more concentrated state. These experiments were also supplemented by the physicochemical characterization of activation solutions as well as prepared BFS dispersions. The outcomes of our experiments are straightforward. The dilution approach was successful for modelling and extrapolation of possible behaviour and stability of BFS dispersions in all used alkaliactivated solutions (sodium hydroxide, sodium silicate and sodium carbonate). We have also observed a different mode of action in the case of sodium hydroxide, where the addition of activation solution increased the zeta potentials from negative values (for zeta potential of slag in water observed average value $(-15.7 \pm 2.2) \mathrm{mV}$ ) to $0 \mathrm{mV}$ and further to positive values. Contrary to this, in the case of sodium silicate and sodium carbonated the addition of AAS into the BFS slag dispersions caused a gradual shift toward more negative values of measured zeta potentials, which is indicating more significant colloidal stabilization of BFS particles in these systems. The results of our work are indicating that the proper technique of sample dispersion and the maintenance of the precise content and ratios of individual components represent crucial parameters managing the stability of alkali-activated materials based on blast furnace slag.

\section{ACKNOWLEDGEMENTS}

\section{The work was supported by project GA20-26896S from the Science Foundation of Czech Republic.}

\section{REFERENCES}

[1] PROVIS, J. L. Geopolymers and other alkali-activated materials: why, how, and what? Materials and Structures. 2014, vol. 47.

[2] PROVIS, J. L. Alkali-activated materials. Cement and Concrete Research. 2018, vol. 114.

[3] KALINA, L., BILEK, BRADOVA, L., TOPOLAR, L. Blast furnace Hybrid Cement with Waste Water Glass Activator: Alkali-Silica Reaction Study. Materials. 2020, vol.13.

[4] BAKHAREV, T., J. G. SANJAYAN AND Y. B. CHENG Resistance of alkali-activated slag concrete to acid attack. Cement and Concrete Research. 2003, vol. 33.

[5] BERNAL, S. A., E. D. RODRÍGUEZ, R. MEJÍA DE GUTIÉRREZ AND J. L. PROVIS Performance of alkaliactivated slag mortars exposed to acids. Journal of Sustainable Cement-Based Materials. 2012, vol. 1.

[6] RASHAD, A. M., Y. BAI, P. A. M. BASHEER, N. C. COLLIER, et al. Chemical and mechanical stability of sodium sulfate activated slag after exposure to elevated temperature. Cement and Concrete Research. 2012, vol. 42.

[7] PROVIS, J. L. and J. S. J. VAN DEVENTER Alkali Activated Materials State-of-the-Art Report, RILEM TC 224AAM. Edtion ed.: Springer, 2014. ISBN 978-94-007-7671-5.

[8] LI, Z., T. LU, X. LIANG, H. DONG, et al. Mechanisms of autogenous shrinkage of alkali-activated slag and fly ash pastes. Cement and Concrete Research. 2020, vol. 135. 
[9] KALINA, L., BILEK, V., BARTONICKOVA, E., KALINA, M., HAJZLER, J., NOVOTNY, R. Doubts over capillary pressure theory in context with drying and autogenous shrinkage of alkali-activated materials. Construction and Building Materials. 2020, vol. 248.

[10] HEWELETT, P. C., LISKA, M. Lea's Chemistry of Cement and Concrete. 5th ed. Oxford, United Kingdom: Elsevier, 2019.

[11] HUNTER, R. J. Zeta potential in colloid science: principles and applications. London: Academic Press, 1988.

[12] KASHANI, A, PROVIS, J. L., QIAO, G. G., VAN DEVENTER, S. J. The interrelationship between surface chemistry and rheology in alkali-activated slag paste. Construction and Building Materials. 2014, vol. 65.

[13] FERRARI, L., J. KAUFMANN, F. WINNEFELD AND J. PLANK Interaction of cement model systems with superplasticizers investigated by atomic force microscopy, zeta potential, and adsorption measurements. $J$ Colloid Interface Sci. 2010, vol. 347.

[14] DUKHIN, A. S. AND P. J. GOETZ Acoustic and electroacoustic spectroscopy for characterizing concentrated dispersions and emulsions. Advances in Colloid and Interface Science. 2001, vol. 92.

[15] VAIL, J.G. Soluble Silicates - Their Properties and Uses. Reinhold Book Corporation. 1952. 